\title{
Mechanism underlying the bio-effects of an electromagnetic field based on the Huang-Ferrell model
}

\author{
D.Y. Geng, G. Hu, L. Wang, N. Jia and F.X. Wang \\ Corresponding author: D.Y. Geng \\ E-mail: gdy_hebut@163.com \\ Genet. Mol. Res. 15 (2): gmr.15028796 \\ Received May 16, 2016 \\ Accepted June 3, 2016 \\ Published June 24, 2016 \\ DOI http://dx.doi.org/10.4238/gmr.15028796
}

Province-Ministry Joint Key Laboratory of Electromagnetic Field and Electrical Apparatus Reliability, Hebei University of Technology, Tianjin, China

\begin{abstract}
To understand the beneficial and harmful bio-effects of extremely low frequency electromagnetic fields, we studied the MAPK/ERK signaling pathway based on the Huang-Ferrell model. The sensitivity analysis method was used to study the influence of the model parameters on the activity of ERK, and to further investigate the key biochemical reactions and proteins. The results of the simulation show that an increase in the reaction rate of MAPK/ERK kinase had little effect on ERK activation and the steady-state molecular number. However, a decrease in the reaction rate of MAPK/ERK kinase significantly affected the trigger time of ERK activation and decreased the steady-state molecular number. Together with the biological significance of ERK activity, our findings indicate that the effects of electromagnetic fields are a result of the decrease in the reaction rate of MAPK/ERK kinase, which eventually determines whether these effects cause physical damage or are beneficial in treatment.
\end{abstract}

Key words: Electromagnetic fields; MAPK/ERK signaling pathway; Sensitivity analysis; Reaction rate 


\section{INTRODUCTION}

With the rapid development of the electrical and information industry, the electromagnetic environment in the living space of humans has become increasingly complex. Power transmission and transformation equipment that use very low frequency electromagnetic fields and communication equipment that use radio frequency electromagnetic radiation form the main component of this environment, because of which the related non-thermal bioeffects of this electromagnetic radiation has become a hot topic of research. Epidemiological studies have shown that exposure to extremely low frequency electromagnetic fields ((ELFEMFs) may be associated with Alzheimer's disease (García et al., 2008), amyotrophic lateral sclerosis (Zhou et al., 2012), childhood asthma (Li et al., 2011) and cancer (Chen et al., 2013), especially childhood leukemia (Schüz, 2011; Grellier et al., 2014) and many other kinds of diseases. However, laboratory studies have not been able to reproduce the results obtained in the epidemiological investigations, but they did show that ELF-EMF (Pesce et al., 2013) plays a beneficial role in wound healing in cancers (Costa et al., 2011) and diseases associated with the nervous system (Di Lazzaro et al., 2013). These data suggest that ELF-EMFs can produce beneficial or harmful pleiotropic effects in organisms or cells. However, there is no universally accepted theory to explain the bio-effects of the pleiotropic effects of electromagnetic fields.

Studying the bio-effects of electromagnetic fields involves the study of the energy coupling between the electromagnetic field and the cell, i.e. how the cells perceive and transmit electromagnetic energy, and then affect the internal biochemical processes such as transcription and translation, and ultimately lead to differences in the apparent biological behavior. Studies on the molecular mechanism underlying the effects of ELF-EMFs show that ELF-EMFs can influence the JAK-STAT pathway, and can lead to phosphorylation of signaling molecules through the action of epidermal growth factor, extracellular signal regulated kinase (ERK1/2) and cAMP-response element binding protein (CREB), and other MAPK pathways. These effects alter the activity of signaling molecules and result in a change in the dynamics of regulatory associations between these molecules. Therefore, cellular signal transduction pathways, especially the signal transduction pathways of ERK in the MAPK pathway, play a crucial role in the process of electromagnetic energy coupling, sensing, and transmitting of the external electromagnetic interference signal.

Cellular signal transduction is in nature a dynamic change in the time domain of signaling molecule activity or concentration caused by complex biochemical reactions in the cell, i.e. cellular signal molecular dynamics. Any small changes in cell receptors or signaling molecules will respond to the change of signal in the form of molecular dynamics, and generate the appropriate output through protein activity change of the upstream change information in the form of complex dynamics, so as to control the dynamic variations of downstream signaling molecules, finally the cells show different biological behavior. Therefore, from the molecular level to study the signal amplification and specific transport properties of signaling molecule concentration change by MAPK pathway, to understand the energy coupling mechanism of diverse biological behavior triggered by weak electromagnetic signals will have a great significance.

\section{MATERIAL AND METHODS}

\section{Modeling theory}

The establishment of a signal transduction pathway model is a hierarchical and abstract 
mode of understanding the real signal transduction network. In general, the signal transduction pathway is simulated by using chemical kinetic equations. Chemical kinetic modeling is the application of mass reaction dynamics to describe all the reactions in a signal pathway, and it is expressed in a general form as

$$
\begin{gathered}
A+B \underset{d}{\stackrel{a}{\rightleftharpoons}} C+D \\
d[A] / d t=-a[A][B]+d[C][D]
\end{gathered}
$$

In Equation 1, $a$ and $d$ are the association and dissociation rate constants; $A$ and $B$ are the substrates; and $C$ and $D$ are the products of the reaction. Equation 2 represents the rate equation of substrate $A$.

The standard Michaelis-Menten (M-M) equation assumes that the last step is irreversible and is a special case of the above reaction; Equation 3 represents one such reaction:

$$
E+S \stackrel{a}{\stackrel{a}{\rightleftharpoons}} C \stackrel{k}{\longrightarrow} E+P
$$

where $a$ and $d$ are the association and dissociation rate constants, and $k$ is the catalytic rate constant; $E$ is an enzyme, $S$ is the substrate to which the enzyme binds to form the complex $C$ $=E S$, and $P$ is the product resulting from the modiðcation of the substrate $S$ due to the binding with the enzyme E. It is generally believed that the generation of the intermediate product $C$ is reversible, but process of production of $P$ through the formation of $C$ is irreversible, as shown in the equation 3. The simplest M-M equation has only one substrate $S$ and one enzyme $E$, and the rate equations for the substrate, product, and intermediate product can be expressed as the following Ordinary Differential Equation (ODE) model

$$
\begin{gathered}
d[S] / d t=-a[E][S]+d[C] \\
d[P] / d t=k[C] \\
d[C] / d t=a[E][S]-d[C]-k[C]=a[E][S]-(d+k)[C]
\end{gathered}
$$

\section{MAPK-ERK pathway model}

ERK not only regulates the behavior of a cell with regard to a variety of normal physiological functions, but also plays an important role in the pathogenesis and pathophysiological processes of a variety of diseases. It also plays a key role in regulation of the behavioral and cognitive functions of humans, such as learning and memory. Although 
the biological response processes associated with the ERK signal transduction pathway are changing, the basic structure is constant. The classical ERK cascade is regulated by reversible phosphorylation of signal transduction molecules, wherein the target protein is activated by phosphorylation of a protein kinase and the subsequent downstream conduction. Simultaneously, a target protein will be deactivated by dephosphorylation under the action of protein phosphatases. When a signaling molecule affecting a variety of proteins is undergoing phosphorylation and dephosphorylation, a complex regulation network of signal transduction is formed to ensure reliable transduction of the upstream information. ERK, in its active state, is controlled by the sequential activation of a cascade reaction in three stages: Ras activates Mos by phosphorylation, and the activation of Mos enables the activation of Mek1 by double phosphorylation. Similarly, activated Mek1 enables the activation of Erk2 by double phosphorylation. This biochemical reaction involving three stages of double phosphorylation ensures the strength and transduction specificity of the upstream stimulus signal. The basic structure of the pathway regulating ERK activity involves a variety of growth factors and processes: Ras $\rightarrow \mathrm{Raf} \rightarrow \mathrm{MEK} 1 / 2 \rightarrow$ ERK1/2 $\rightarrow$ cell growth, development, division, and differentiation. The basic structure of the ERK signal transduction pathway is shown in Figure 1.

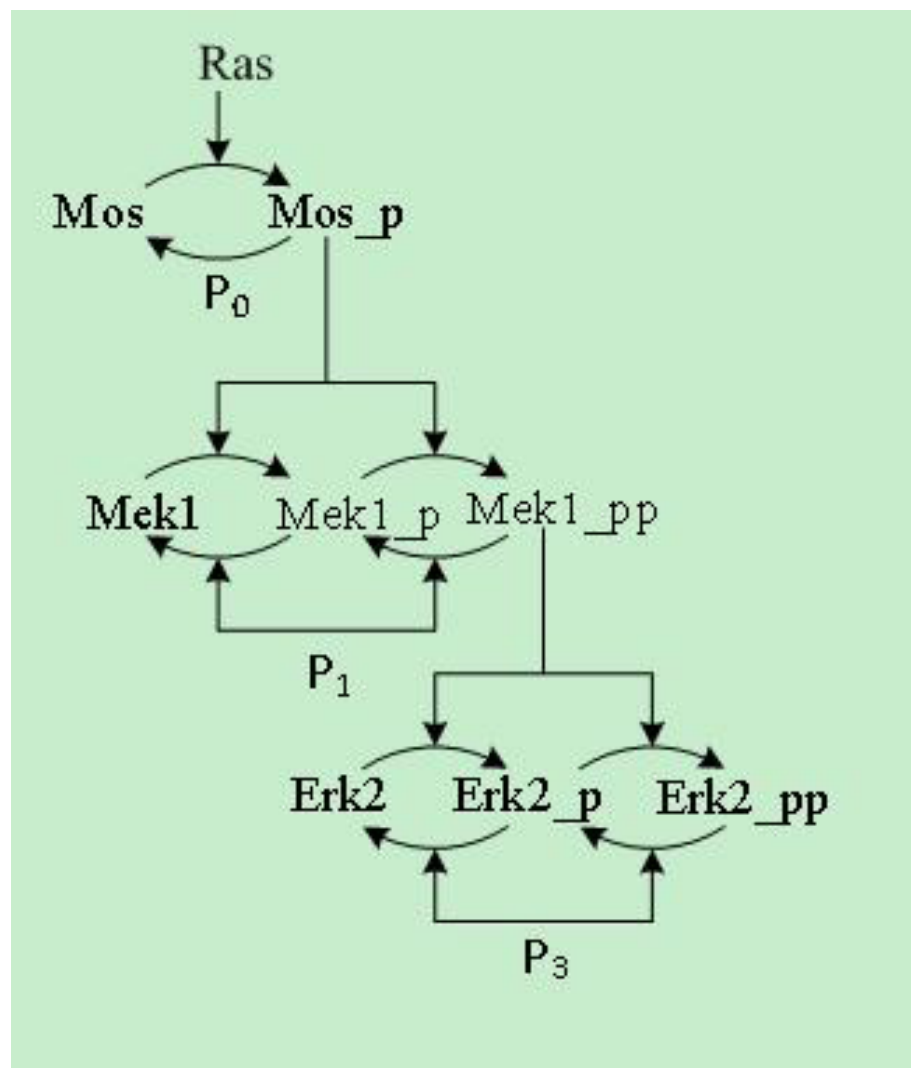

Figure 1. Schematic representation of the 3-level cascade structure of the ERK signaling pathway. 
In this study, we performed our investigation based on the Huang-Ferrell model (Huang and Ferrell, 1996). We now provide the entire set of reactions for the MAPK cascade represented in Figure 1:

$$
\begin{aligned}
& \text { Ras }+ \text { Mos } \underset{k 2-1}{\stackrel{k 1-1}{\rightleftharpoons}} E_{1} / \text { Mos } \stackrel{k 1-2}{\longrightarrow} \text { Mos } \_p+\text { Ras } \\
& P_{0}+M o s_{-} p \underset{k 2-3}{\stackrel{k 1-3}{\rightleftharpoons}} E_{2} / M o s_{-} p \stackrel{k 1-4}{\longrightarrow} M o s+P_{0} \\
& \text { Mos _ } p+\operatorname{Mek} 1 \stackrel{k 1-5}{\underset{k 2-5}{\rightleftharpoons}} p_{-} \text {Mos } / \text { Mek } 1 \stackrel{k 1-6}{\longrightarrow} \text { Mek_ } p+\text { Mos_p } \\
& M e k 1_{-} p+P_{1} \underset{k 2-7}{\stackrel{k 1-7}{\rightleftharpoons}} P_{1} / p_{-} \operatorname{Mek} 1 \stackrel{k 1-8}{\longrightarrow} \operatorname{Mek} 1+P_{1} \\
& \text { Mos } s_{-} p+M e k 1_{-} p \stackrel{k 1-9}{\underset{k 2-9}{\rightleftharpoons}} p_{-} M o s / p_{-} M e k 1 \stackrel{k 1-10}{\longrightarrow} M e k 1_{-} p p+M o s_{-} p \\
& \text { Mek_ } p p+P_{1} \underset{k 2-11}{\stackrel{k 1-11}{\rightleftharpoons}} P_{2} / p p_{-} M e k 1 \stackrel{k 1-12}{\longrightarrow} M e k 1_{-} p+P_{1}
\end{aligned}
$$

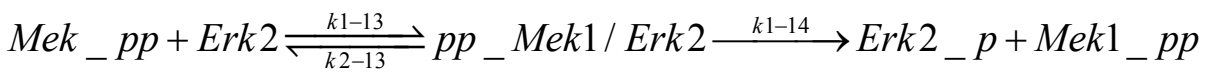

$$
\begin{aligned}
& E r k 22_{-} p+P_{2} \underset{k 2-15}{\stackrel{k 1-15}{\rightleftharpoons}} P_{2} / p_{-} E r k 2 \stackrel{k 1-16}{\longrightarrow} \operatorname{Erk} 2+P_{2}
\end{aligned}
$$

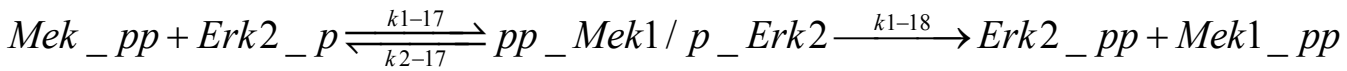

$$
\begin{aligned}
& E r k 2_{-} p p+P_{2} \underset{k 2-19}{\stackrel{k 1-19}{\rightleftharpoons}} P_{2} / p p_{-} E r k 2 \stackrel{k 1-20}{\longrightarrow} \operatorname{Erk} 2_{-} p+P_{2}
\end{aligned}
$$

where $\mathrm{k} 1-(2 \mathrm{j}-1)$ is the association rate constant, and $\mathrm{k} 2-(2 \mathrm{j}-1)$ and $\mathrm{k} 1-(2 \mathrm{j})$ are the dissociation rate constants. Here we set $\mathrm{j}=1, \ldots 10$. For example, $\mathrm{k} 1-1$ is the association rate constant of E1/ Mos, and k2-1 and K1-2 are the dissociation rate constants of E1/Mos; E1/Mos is a complex of Ras and Mos.

We achieved the input and output of the dynamics of the relationship between the protein molecules by using MATLAB software ODE15s solver programming, and achieved system sensitivity analysis by using the COPASI software partial sensitivity analysis method.

\section{RESULTS}

\section{Sensitivity analysis to identify the key model parameters}

Sensitivity analysis is a commonly used in the study of signal transduction pathways to analyze the effect of changes in model parameters on the system response and to identify the system response or protein molecules, which make impact on the entire system greater in system model. In addition, sensitivity analysis is also used to evaluate the robustness of a model. Local parameter sensitivity analysis is the study of the effects of a change in a particular parameter and its effects on the output of the model (e.g., Erk2_pp concentration). This method is used to examine the stability of a system in response to small changes in the model parameters. For the key parameters, a small disturbance is very likely to cause significant changes in the system variables, and different parameters have different sensitivities. The relationship between a 
specific parameter $\mathrm{P}$ and a sensitivity parameter $(S C)$ of a system variable $\mathrm{V}$ can be expressed by the following equation:

$$
S C_{P}^{V}=\frac{\delta V / V}{\delta P / P}
$$

in which $V$ represents the output of the model (e.g. Erk2_pp concentration), $d V$ represents the change in parameter $P$ caused by the change in the system variable $V . P$ represents a particular parameter. $d P$ represents a small change in parameter $\mathrm{P}$ made while keeping the other parameters constant. Sensitivity analysis is performed from the perspective of quantitative analysis to study how changes in the initial concentration or kinetic parameters within a certain range affect the model output (specific set of changes in the concentration or model of the overall state of the change) (Qi, 2014). Sensitivity analysis has significance in the identification of the key control points of any biological network. Some of the components of biological networks under small perturbations show high sensitivity, usually represent "brittle" (the antonym of "robustness"). Changes in biological functions caused by changes in these parameters may play a very important role in the occurrence and development of a disease, and can be used as a potential target for drug discovery and development (Luan et al., 2007). At present, the commonly used sensitivity analysis methods include a method in which only one factor is changed at one time, the local sensitivity analysis method, scatter plot method, regression analysis, and the variance-based approach and screening method. In addition, many modeling software such as COPASI and SB ToolBox can now perform sensitivity analysis. We established a model of sensitivity analysis by using COSPASI, which is a software tool used for editing, simulating, and analyzing models of biochemical reaction networks. The software can be obtained from http://copasi.org/. COPASI also allows the calculation of the sensitivities of the model with respect to various parameters. Sensitivity is generally a measure for how much a speciðc "observable" variable (this means any number that can be obtained by numerical analysis of the model) changes when a given parameter is changed. In COPASI, it is possible to calculate whole arrays of sensitivities for lists of "observables" with respect to lists of parameters. The settings for the sensitivity calculations can be found under "Tasks$>$ Sensitivities". Let us look at an example in Figure 2:

For "Subtask", choose "Steady State". This means that you are interested in the sensitivities of a result of a steady state calculation.

For "Function", choose "Non-Constant Concentration of Species". "Function" here indicates the observables, i.e. the values of which you want to know the sensitivities. We want to calculate the sensitivities of the steady state concentrations.

Set "Variable" to "Local Parameter Values". Local parameters are all kinetic parameters that are directly speciðed for the reactions in the model.

To start the calculation, just click on the run button; a part of the results obtained are shown in Figure 3.

The results are displayed as color-coded tables of numbers. Therefore, we collected and analyzed the data to obtain a histogram of sensitivity analysis shown in Figure 4. In fact, most of the parameters produced a very small change and hence were not listed in the histogram. 


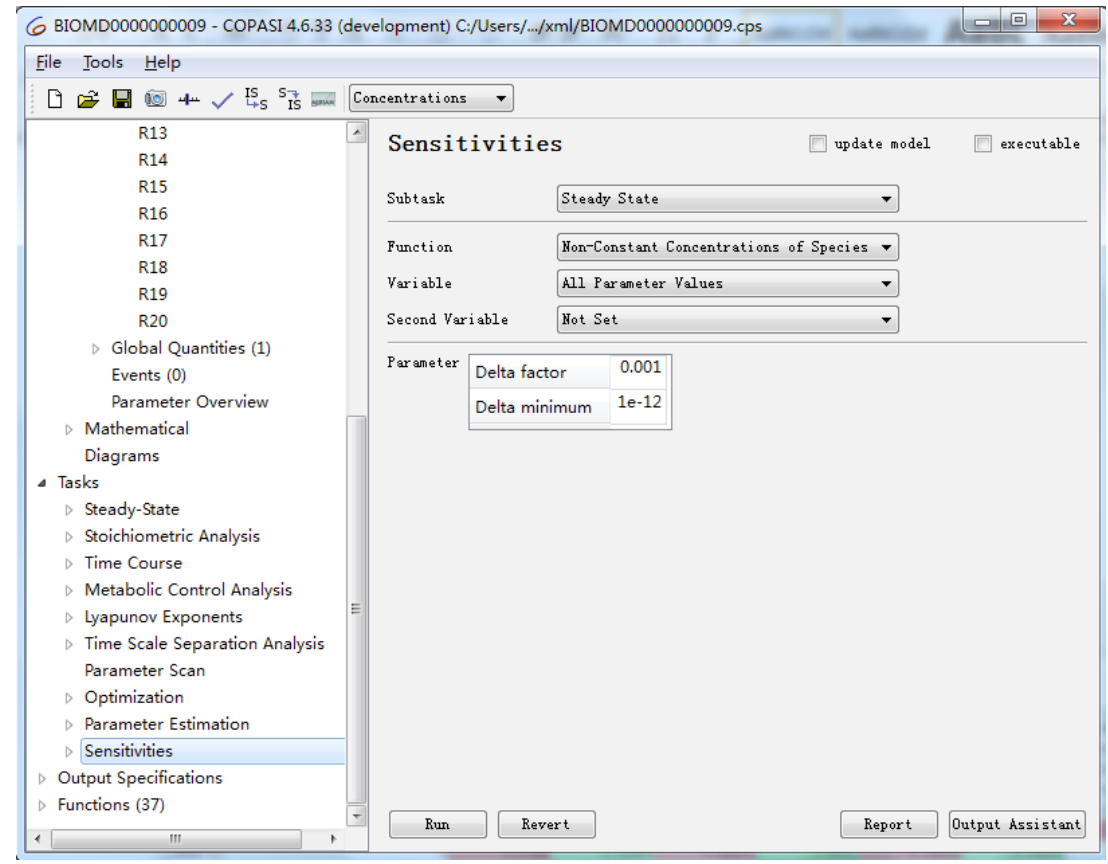

Figure 2. Sensitivity analysis dialog.

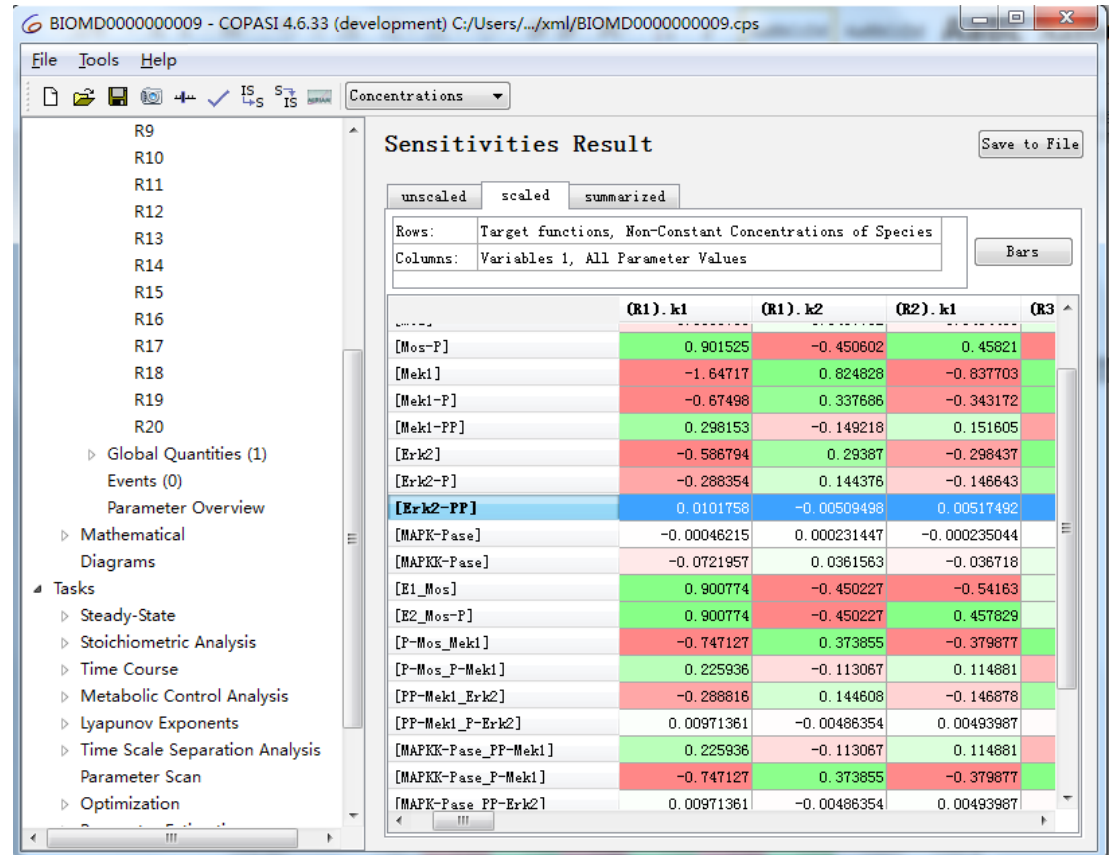

Figure 3. Part of the result of the sensitivity analysis. 


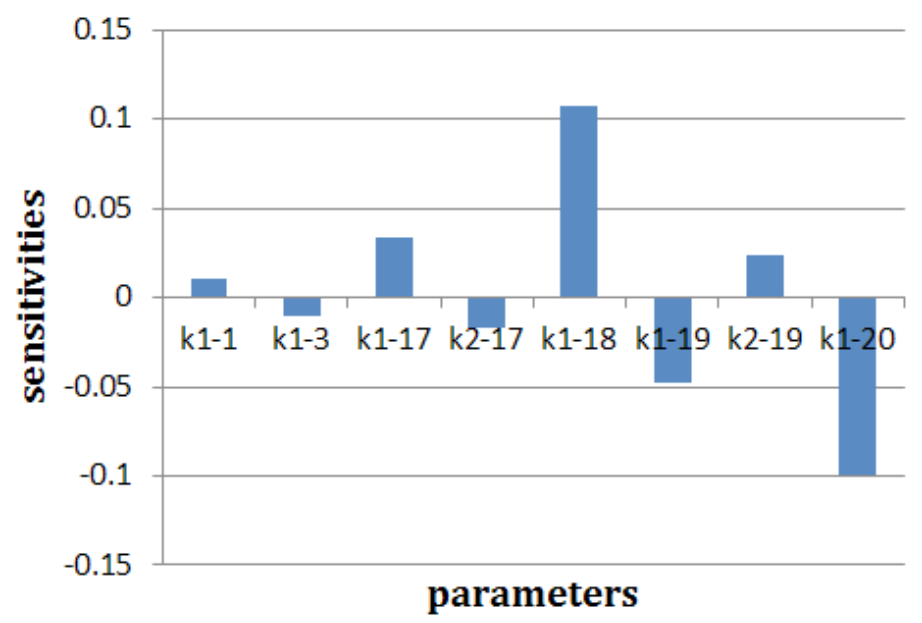

Figure 4. Histogram of sensitivity analysis for the output Erk2_pp.

Here, sensitivity analysis was carried out on various biochemical reaction rate constants, which affect the output Erk2_pp. From Figure 4, we can see that k1-18 is a key parameter, which is the most sensitive for the output Erk2_pp. k1-18 is the dissociation reaction rate constant of pp_Mek1/p_Erk2, and pp_Mek1/p_Erk2 is the complex of Mek1_pp and Erk2 pp. Therefore, a change in the value of k1-18 will significantly affect the concentration of Erk2_pp, and then directly affect the activity or concentration of ERK.

\section{Effects of sensitive parameters on ERK activity}

We studied the key sensitive parameter, k1-18, which influences the output of Erk2 pp, and its effect on Erk2_pp is shown in Figure 5.
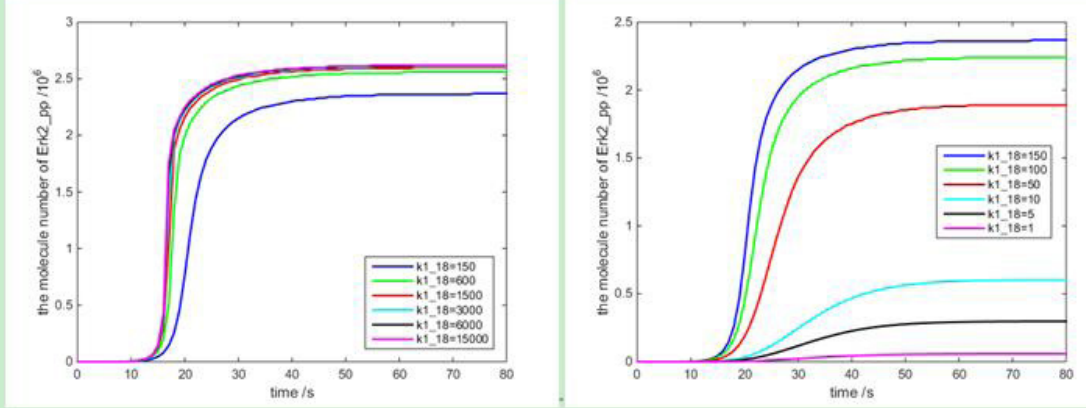

(a) The value of $k 1-18$ increases gradually $\cdots$ (b) The value of $k 1-18$ decreases gradually

Figure 5. The influence of the change in k1-18 on the output Erk2 pp of the model. In Figure 5a, we use the blue, green, red, cyan, black, and magenta, six color curves correspond to the number of the activated molecules of Erk2_pp with a gradual increase in k1-18 $(150,600,1500,3000,6000$, and 15,000, respectively). Similarly, in Figure 5b, the blue, green, red, cyan, black and magenta, six color curves correspond to the number of the activated molecules of Erk2_pp with a gradual decrease in k1-18 (150, 100, 50, 10, 5, and 1, respectively). 
Figure 5a shows that with the gradual increase in k1-18, the time of activation of Erk2_pp and the steady-state molecular number of Erk2_pp both change in the beginning, but the final change at the end with respect to the value at the beginning of the reaction is only small. Figure $5 \mathrm{~b}$ shows that with the gradual decrease in k1-18, the time of activation of Erk2_pp gradually increased and the steady-state molecular number of Erk2 pp decreased. At the same time, Erk2_pp goes into a transient state of activation from the initial state of sustained activation. Thus, the change in protein activity with time can result in a change of system function and the cellular signal transduction network characteristics.

The bio-effects of electromagnetic experiments have shown that ELF-EMFs significantly affect the activity of ERK. Therefore, we established a model of the MAPK/ERK signaling pathway to identify the factors that significantly influence the output Erk2 pp of the upstream signal transduction using sensitivity analysis methods. In addition, the proteins involved in the reaction that determines the change in the activity of ERK were identified. Further, by changing the key parameters (the parameters that significantly affect Erk2_pp), we discovered that ERK activity is closely related to the decrease in the reaction rate rather than to the increase in the reaction rate in the biochemical reaction. For example, the change in k118 explained the fundamental cause of the pleiotropic bio-effects of electromagnetic fields is the resulting change in the strength of the interaction between the two proteins, which further alters the biological behavior of cells.

In fact, the cell can be through the network of signal transduction to encode and transport the time varying information of concentration, activity, modification status and position of the effected signaling molecule to generate the dynamics change in the abundance of frequency, amplitude and duration (Purvis and Lahav, 2013) of the downstream signal molecules, and then induce the cells to produce different biological behavior (Purvis et al., 2012), and obtain the diversity of bio-effects.

Studies have shown that ERK1/2 activity was abnormally high in breast cancer specimens, and that the expression of phosphorylated ERK in lymph node metastasis was significantly higher than that in the matched primary tumors. In addition, ERK1/2 in liver cancer, head and neck squamous cell carcinoma, prostate cancer, and other tumor tissues showed abnormal expression or enhanced activity (Branca et al., 2004; Purvis et al., 2012; Purvis and Lahav, 2013), suggesting a close relationship between abnormal ERK regulation and the occurrence and development of tumors. Based on our findings, the gradual decrease in the parameter k1-18 can significantly alter the activation molecular curve of Erk2_pp, and ultimately affect the physiological behavior of cells, which may be used as a target for the treatment of cancer.

\section{DISCUSSION}

According to the simulation outcome of the MAPK signaling pathway model, we found that ERK activity is closely related to the decrease in the reaction rate rather than to increase in the reaction rate in the biochemical reaction. In addition, the parameter sensitivity analysis showed that ERK activity was significantly affected by ERK kinase, MAPK/ERK kinase, and Ras protein. Therefore, the main reason for the change in ERK activity induced by electromagnetic fields is the result of the biochemical reaction rate for reactions involving the above three kinds of kinases and protein. Moreover, as a signaling molecule, ERK has different dynamic characteristics with regard to its activity, and ERK activity is an important factor determining cell behavior. 


\section{Conflicts of interest}

The authors declare no conflicts of interest.

\section{ACKNOWLEDGMENTS}

Research supported by the National Natural Science Foundation of China (Grant \#51507047), the National Natural Science Foundation of China (Grant \#61571180) and the Natural Science Foundation of Hebei Province, China (Grant \#2016202128).

\section{REFERENCES}

Branca M, Ciotti M, Santini D, Bonito LD, et al.; HPV-Pathogen ISS Study Group (2004). Activation of the ERK/MAP kinase pathway in cervical intraepithelial neoplasia is related to grade of the lesion but not to high-risk human papillomavirus, virus clearance, or prognosis in cervical cancer. Am. J. Clin. Pathol. 122: 902-911 http://dx.doi. org/902-911.10.1309/VQXFT880JXC7QD2W. http://dx.doi.org/10.1309/VQXFT880JXC7QD2W

Chen Q, Lang L, Wu W, Xu G, et al. (2013). A meta-analysis on the relationship between exposure to ELF-EMFs and the risk of female breast cancer. PLoS One 8: e69272 http://dx.doi.org/10.1371/journal.pone.0069272.

Costa FP, de Oliveira AC, Meirelles R, Machado MC, et al. (2011). Treatment of advanced hepatocellular carcinoma with very low levels of amplitude-modulated electromagnetic fields. Br. J. Cancer 105: 640-648 http://dx.doi. org/10.1038/bjc.2011.292.

Di Lazzaro V, Capone F, Apollonio F, Borea PA, et al. (2013). A consensus panel review of central nervous system effects of the exposure to low-intensity extremely low-frequency magnetic fields. Brain Stimulat. 6: 469-476 http://dx.doi. org/10.1016/j.brs.2013.01.004.

García AM, Sisternas A and Hoyos SP (2008). Occupational exposure to extremely low frequency electric and magnetic fields and Alzheimer disease: a meta-analysis. Int. J. Epidemiol. 37: 329-340 http://dx.doi.org/10.1093/ije/dym295.

Grellier J, Ravazzani P and Cardis E (2014). Potential health impacts of residential exposures to extremely low frequency magnetic fields in Europe. Environ. Int. 62: 55-63 http://dx.doi.org/10.1016/j.envint.2013.09.017.

Huang CY and Ferrell JE Jr (1996). Ultrasensitivity in the mitogen-activated protein kinase cascade. Proc. Natl. Acad. Sci. USA 93: 10078-10083. http://dx.doi.org/10.1073/pnas.93.19.10078

Li DK, Chen H and Odouli R (2011). Maternal exposure to magnetic fields during pregnancy in relation to the risk of asthma in offspring. Arch. Pediatr. Adolesc. Med. 165: 945-950 http://dx.doi.org/10.1001/archpediatrics.2011.135.

Luan D, Zai M and Varner JD (2007). Computationally derived points of fragility of a human cascade are consistent with current therapeutic strategies. PLoS Comput. Biol. 3: e142 http://dx.doi.org/10.1371/journal.pcbi.0030142.

Pesce M, Patruno A, Speranza L and Reale M (2013). Extremely low frequency electromagnetic field and wound healing: implication of cytokines as biological mediators. Eur. Cytokine Netw. 24: 1-10 10.1684/ecn.2013.0332.

Purvis JE and Lahav G (2013). Encoding and decoding cellular information through signaling dynamics. Cell 152: 945 956 http://dx.doi.org/10.1016/j.cell.2013.02.005.

Purvis JE, Karhohs KW, Mock C, et al. (2012). p53 dynamics control cell fate [J]. Science 336: 1440-1444. 10. http:// dx.doi.org/1126/science. 1218351

Qi YF (2014). Modeling the signal transduction of IFN- $\gamma$ and IL-6 pathways and eluciding the mechanism of anti-cancer drug action on cell apoptosis via system biology methods [D]. Northeast Normal University, Jilin.

Schüz J (2011). Exposure to extremely low-frequency magnetic fields and the risk of childhood cancer: update of the epidemiological evidence. Prog. Biophys. Mol. Biol. 107: 339-342 http://dx.doi.org/10.1016/j. pbiomolbio.2011.09.008.

Zhou H, Chen G, Chen C, Yu Y, et al. (2012). Association between extremely low-frequency electromagnetic fields occupations and amyotrophic lateral sclerosis: a meta-analysis. PLoS One 7: e48354 http://dx.doi.org/10.1371/ journal.pone.0048354. 